\title{
Ab Initio Molecular Orbital Calculations for the Double-Stranded Helices of Poly(methyl methacrylate) Monomer Model
}

\author{
Hiroshi Kusanagi, Yozo Chatani, ${ }^{*}$ and Hiroyuki Tadokoro* \\ Unitika Research Laboratories Inc., Uji, Kyoto 611, Japan \\ * Department of Macromolecular Science, Faculty of Science, \\ Osaka University, Toyonaka, Osaka 560, Japan
}

(Received March 15, 1996)

\begin{abstract}
KEY WORDS Ab Initio Calculation / Double-Stranded Helix / Poly(methyl methacrylate) / Monomer / Stabilizing Energy /
\end{abstract}

In a previous paper, $a b$ initio molecular orbital (MO) calculations were made to evaluate the energetical stability of the double-stranded helix of poly(ethyleneimine) $\left(-\left(\mathrm{NHCH}_{2} \mathrm{CH}_{2}\right)_{n}-\right.$ : PEI) oligomers, a model compound for polymer double-stranded helix. ${ }^{1}$ And the doublestranded helix of PEI was found to be stabilized with the formation of $\mathrm{N}-\mathrm{H} \cdots \mathrm{N}$ hydrogen bonding between the two polymer chains in the double-strand, as same as in DNA double-stranded helices. ${ }^{2,3}$ That is, calculated total MO energy of double-stranded helix for pentamer gave the stabilizing energy of $\Delta E=-2.71 \mathrm{kcal} \mathrm{mol}^{-1}$ of monomer unit, in comparison with twice of the isolated single helix.

Isotactic poly(methyl methacrylate) (it-PMMA) has also known to take the $(10 / 1)$ double-stranded helix in the crystal. ${ }^{4}$ It would seem to be important to clarify the structural reasons why it-PMMA does form the doublestranded helix, although there is no strong interactions between the two helices, such as, for example, hydrogen bonding in $\mathrm{DNA}^{2,3}$ and PEI. ${ }^{1}$ In other words, a theoretical proof that the double-stranded helix structure of it-PMMA is correct is asked for. However, there are no studies on the theoretical calculation of the it-PMMA double-stranded helix structure determined by X-ray analysis. Ab initio MO method is the most reliable theoretical calculation procedure. Therefore, this method has been also applied to the double-stranded helices of itPMMA.

We are aiming to evaluate the stabilizing mechanism of double-stranded helix of it-PMMA polymer itself in terms of the $a b$ initio MO calculations. But, the $a b$ initio MO method has a serious limitation on the number of involving atoms as stated in the next section. At the first stage of our purpose, in this study, ab intio MO calculations $^{5}$ were made on the it-PMMA monomer with the same geometry of the double-stranded helix.

\section{METHOD}

$A b$ initio molecular orbital calculations were carried out with using the version of the Hondo- 8 system of programs; applicable maximal number of atoms is 128 . The $4-31 \mathrm{G}$ basis set was adopted, and ab initio SCF-MO method was used as the calculation procedure. For the more accurate calculation of intermolecular interaction, the electron correlation correction to the energy of closed shell RHF wavefunctions was calculated by means of the 2nd order Moeller-Plesset (MP2) perturbation theory. ${ }^{6}$ The present Hondo- 8 program has been used for MP2 calculations involving up to 250 basis functions. Therefore, we can calculate on the monomer model (including 34 atoms and 166 basis functions) of double-stranded helix in terms of the MO method with MP2 perturbation, although the trimer model (including 94 atoms and 490 basis functions) is treated with the MO method without MP2 perturbation.

The smallest model of double-stranded helix of itPMMA monomer $\left(\mathrm{H}-\mathrm{CH}_{2}-\mathrm{C}\left(\mathrm{COOCH}_{3}\right)\left(\mathrm{CH}_{3}\right)-\mathrm{H}: 17\right.$ atoms), which involves 166 basis functions, was constructed as a model compound for polymer, using the structural parameters determined by X-ray analysis as shown in Figure 1. Since the double-stranded helix of
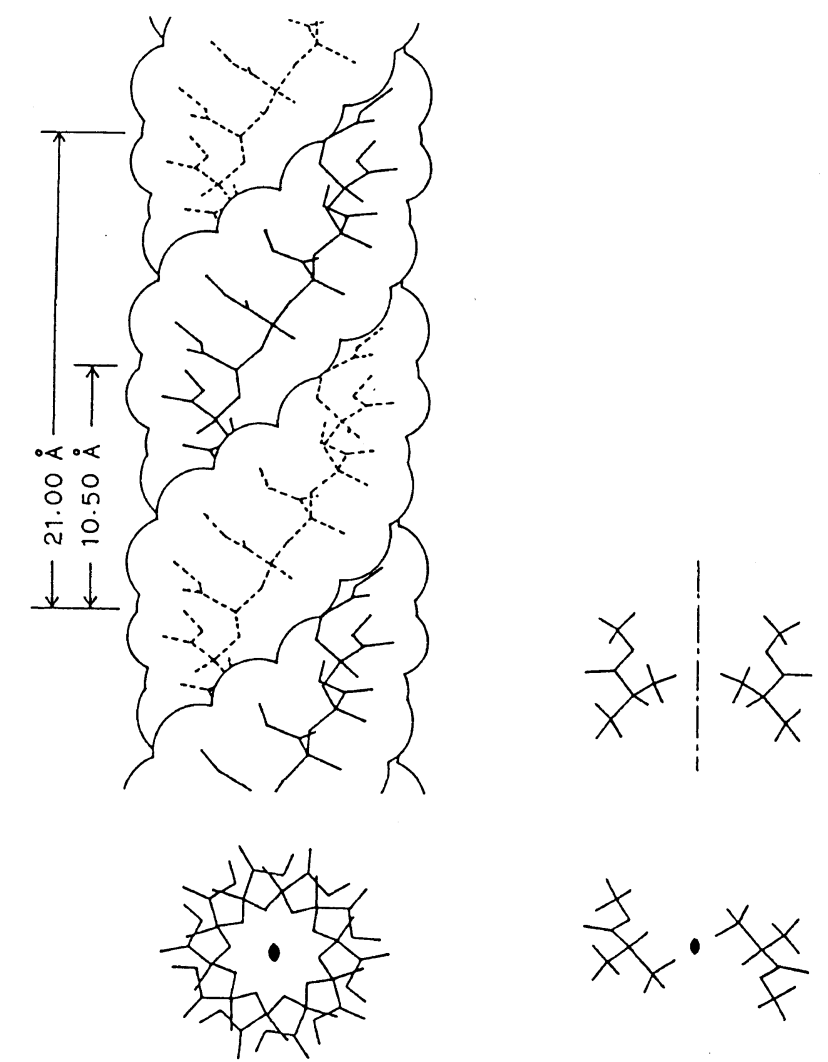

(a)

(b)

Figure 1. Double-stranded helical structure models for (a) polymer and (b) monomer of it-PMMA. 
it-PMMA has the two-fold rotation axis coinciding to the helix axis, i.e., $\mathrm{C}_{2}$-symmetry, each single helix chain constituting the double-stranded helix has the same geometry (denoted to $A$ ). For the structure of doublestranded helix model, total energies $E(A \cdots A)$ were calculated with 4-31G basis set with MP2 method. On the other hand, total energy $E(A)$ of the single helix model was calculated using the one half geometry of the structure. The interaction energy $\Delta E$ (=stabilizing energy) between the two molecular chains in the doublestranded helix, in other words, the complex formation energy can be calculated as follows, ${ }^{7}$

$$
\Delta E=[E(A \cdots A)-2 E(A)] / 2
$$

IBM computer work station was used for the calculation.

\section{RESULTS AND DISCUSSION}

Table I summarizes the calculation results of the total energies $E(A \cdots A), E(A)$, and stabilizing energy $\Delta E$.

At first, the RHF calculations without the MP2 method was performed in the same manner for the double-stranded helix of PEI. In this case, the doublestranded helix model of monomers (molecular complex) became unstable, because of $\Delta E=0.27 \mathrm{kcal} \mathrm{mol}^{-1}$ (positive value). Next, the RHF calculation was carried out with a large double-stranded helix model composed of two trimers. But the larger model also gives a positive value of $\Delta E=1.70 \mathrm{kcal} \mathrm{mol}^{-1}$ of monomer. Therefore, the calculations including the electron correlation correction with MP2 method was carried out. As the result, for the double-stranded helix of it-PMMA monomer, the stabilizing energy of $\Delta E=-0.53 \mathrm{kcal} \mathrm{mol}^{-1}$ was produced from the electron correlation correction, so that total stabilizing energy was calculated to be $\Delta E=$ $-0.26 \mathrm{kcal} \mathrm{mol}^{-1}$. This value of $-0.26 \mathrm{kcal} \mathrm{mol}^{-1}$ is very small as compared with $\Delta E=-1.45 \mathrm{kcal} \mathrm{mol}^{-1}$ of stabilizing energy by the hydrogen bonding for PEI dimer model. But, both of the values are reasonable from the difference between the van der Waals interactions and hydrogen bonding interactions for it-PMMA and PEI, respectively. Therefore, it would be meaningful that the steric structure of the double-stranded helix of it-PMMA was found, for the first time, to be stable in the $a b$ initio MO calculation.

The present result indicates that the electron correlation collection to intermolecular interactions should be an extremely important and dominant factor for the van der Waals interactions. For the double-stranded helix some sliced sectional planes normal to the helix axis are shown in Figure 2, presented in terms of the van der Waals molecular model. ${ }^{4}$ A very good van der Waals fitting between the two intertwined chains is can be seen. This van der Waals fitting based on the experience was confirmed for the first time by the $a b$ initio MO calculations based on the basic theory.

The present calculation on the monomer model is not
Table I. Total energy $(E)$ and stabilizing enegy $(\Delta E)$ of double-stranded helix of it-PMMA monomer model

\begin{tabular}{|c|c|c|c|c|}
\hline & $E(A \cdots A)$ & $E(A)$ & $\Delta E^{\mathrm{a}}$ & $\Delta E^{\mathrm{a}}$ \\
\hline & $\mathrm{au}$ & $\mathrm{au}$ & $\mathrm{au}$ & $\mathrm{kcal} \mathrm{mol}^{-1}$ \\
\hline $\mathrm{RHF}$ & -668.74266 & -344.37176 & 0.00043 & 0.269 \\
\hline MP2 energy & -1.44904 & -0.72368 & -0.00084 & -0.526 \\
\hline Total energy & -690.19170 & -345.09544 & -0.00041 & -0.257 \\
\hline
\end{tabular}

${ }^{\mathrm{a}} \Delta E=[E(A \cdots A)-2 E(A)] / 2$.

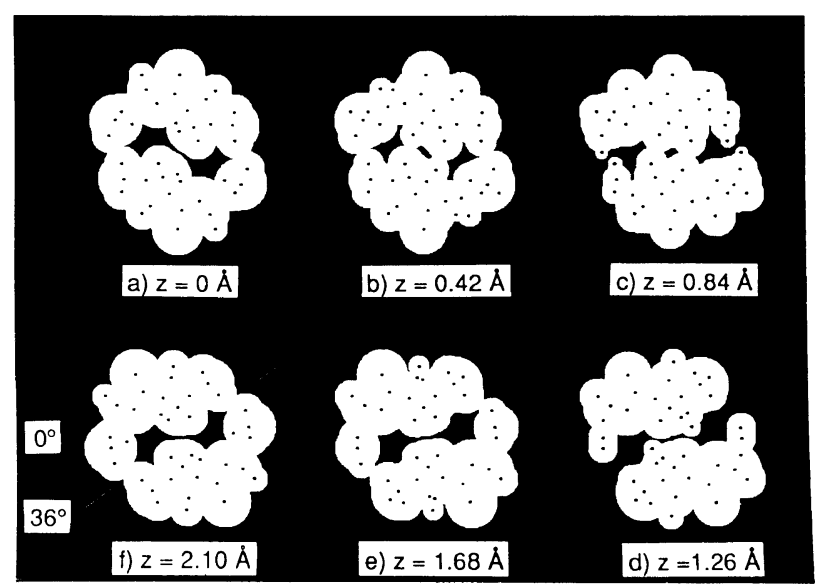

Figure 2. Sliced sectional planes of double-stranded helix, normal to the helix axis, of it-PMMA. (a) and (f) are separated by $2.1 \AA$ corresponding to the displacement of one monomer.

enough, because it is neglect in this case that the polymer effect, i.e., the long range interaction energies along the helix axis between the intertwined two chains may contribute to the increasing in this stabilizing energy. ${ }^{8}$ The stabilizing energy, $\Delta E=-0.26 \mathrm{kcal} \mathrm{mol}^{-1}$, of monomer model is expected to become large for some oligomer models. Therefore, the further investigation on large oligomer models is in progress.

Acknowledgments. This work is based in part on results obtained from the MOTECC Package.

\section{REFERENCES}

1. H. Kusanagi, Polym. J., 28, 362 (1996).

2. J. D. Watson and H. C. Crick, Nature (London), 171, 737 (1953)

3. R. Lagrige, H. R. Wilson, C. W. Hooper, and M. H. F. Wilkins, J. Mol. Biol., 2, 19 (1960).

4. H. Kusanagi, Y. Chatani, and H. Tadokoro, Polymer, 35, 2028 (1994).

5. I. Ando, "Solid NMR of Polymer," Kodansya, Tokyo, 1994, p 24.

6. J. Yonezawa, T. Nagata, H. Kato, S. Imamura, and K. Morokuma, "Introduction to Quantam Chemistry," 3rd ed, Kagaku-Dojin, Kyoto, 1985, p 402.

7. J. Yonezawa, T. Nagata, H. Kato, S. Imamura, and K. Morokuma, "Introduction to Quantam Chemistry," 3rd ed, Kagaku-Dojin, Kyoto, 1985, p 610.

8. J. Yonezawa, T. Nagata, H. Kato, S. Imamura, and K. Morokuma, "Introduction to Quantum Chemistry," 3rd ed, Kagaku-Dojin, Kyoto, 1985, p 568. 